\title{
Efficient Adaptive Strategy for Solving Inverse Problems
}

\author{
M. Paszyński ${ }^{1}$, B. Barabasz ${ }^{2}$, and R. Schaefer ${ }^{1}$ \\ ${ }^{1}$ Department of Computer Science \\ 2 Department of Modeling and Information Technology \\ AGH University of Science and Technology, \\ Al. Mickiewicza 30, 30-059 Cracow, Poland, \\ \{paszynsk, schaefer\} @agh.edu.pl, \\ barabasz@metal.agh.edu.pl \\ http://home.agh.edu.pl/ paszynsk
}

\begin{abstract}
The paper describes the strategy for efficient solving of difficult inverse problems, utilizing Finite Element Method (FEM) as a direct problem solver. The strategy consists of finding an optimal balance between the accuracy of global optimization method and the accuracy of an $h p$-adaptive FEM used for the multiple solving of the direct problem. The crucial relation among errors was found for the objective function being the energy of the system defining the direct problem. The strategy was applied for searching the thermal expansion coefficient (CTE) parameter in the Step-and-flash Imprint Lithography (SFIL) process.
\end{abstract}

Keywords: Inverse problems, Finite Element Method, $h p$ adaptivity, Molecular Statics.

\section{Introduction}

Inverse parametric problems belong to the group of heaviest computational tasks. Their solution require a sequence of direct problem solutions, e.g. obtained by Finite Element Method (FEM), thus the accuracy of the inverse problem solution is limited by the accuracy of the direct problem solution. We utilize the fully automatic $h p$ FEM codes 6.3] generating a sequence of computational meshes delivering exponential convergence of the numerical error with respect to the mesh size for solving direct problems. Using the maximum accuracy for the direct problem solve by each iteration of inverse solver leads to needles computational costs (see e.g. 4]). A better strategy is to balance dynamically the accuracy of both iterations.

However, to be able to execute such strategy we need to relate the error of optimization method defined as the uncorrectness of objective function value with the FEM solution error. We propose such relation and the detailed error balance strategy for the objective being the energy of the system that is described by the simple problem. 
The strategy is tested on the Step-and-Flash Impring Lithography (SFIL) simulations. The objective of the inverse analysis is to find value of the thermal expansion coefficient enforcing shrinkage of the feature well comparable with experimental data. The energy used for the error estimation of the objective function was obtained from the experimental data and static molecular model calculations [5].

\section{The Automatic $h p$ Adaptive Finite Element Method}

Sequential and parallel 3D $h p$ adaptive FEM codes [6], 3] generate in fully automatic mode a sequence of $h p \mathrm{FE}$ meshes providing exponential convergence of the numerical error with respect to size of the mesh (number of degrees of freedom, CPU time). Given an initial mesh, called the coarse mesh, presented
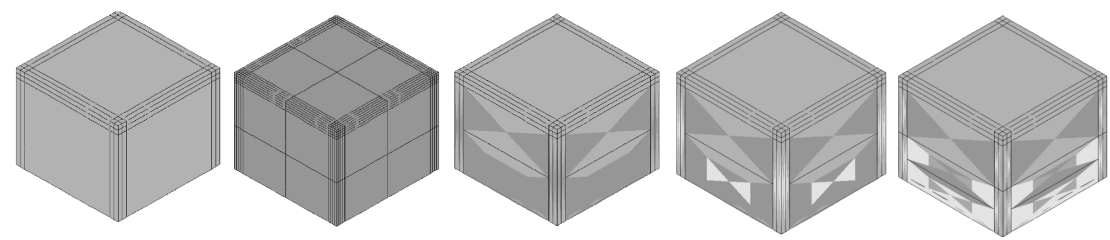

Fig. 1. The coarse mesh with $p=2$ and fine mesh with $p=3$ on all elements edges, faces, and interiors. The optimal meshes after the first, second and third iterations. Various colors denote various polynomial orders of approximation.

on the first picture in Fig. 1, with polynomial orders of approximations $p=2$ on elements edges, faces and interiors, we first perform global $h p$ refinement to produce the fine mesh presented on the second picture in Fig. 1, by breaking each element into 8 son elements, and increasing the polynomial order of approximation by one. The direct problem is solved on the coarse and on the fine mesh. The energy norm (see e.g. [1]) difference between coarse and fine mesh solutions is then utilized to estimate relative errors over coarse mesh elements. The optimal refinements are then selected and performed for coarse mesh elements with high relative errors. The coarse mesh elements can be broken into smaller son elements (this procedure is called $h$ refinement) or the polynomial order of approximation can be increased on element edges, faces or interiors (this procedure is called $p$ refinement), or both (this is called $h p$ refinement). For each finite element from the coarse mesh we consider locally several possible $h, p$ or $h p$ refinements. For each finite element the refinement strategy providing maximum error decrease rate is selected. The error decrease rate

$$
\text { rate }=\frac{\left\|u_{h / 2, p+1}-u_{h p}\right\|-\left\|u_{h / 2, p+1}-w_{h p}\right\|}{n r d o f_{\text {added }}} .
$$

is defined as relative error estimation in energy norm divided by number of degrees of freedom added. Here $u_{h p}$ stands for the coarse mesh solution, $u_{h / 2, p+1}$ 
for the fine mesh solution and $w_{h p}$ is the solution corresponding to proposed refinement strategy, obtained by the projection based interpolation technique [2]. The optimal mesh generated in such a way becomes a coarse mesh for the next iteration, and the entire procedure is repeated as long as the global relative error estimation is larger then the required accuracy of the solution (see [6] for more details). The sequence of optimal meshes generated by automatic $h p$-adaptive code from the coarse mesh is presented on third, fourth and fifth pictures in Figure 1. The relative error of the solution goes down from $15 \%$ to $5 \%$.

\section{The Relation Between the Objective Function Error and the Finite Element Method Error}

We assume the direct problem is modeled by the abstract variational equation

$$
\left\{\begin{array}{l}
u \in u_{0}+V \\
b(u, v)=l(v) \forall v \in V
\end{array}\right.
$$

where $u_{0}$ is the lift of the Dirichlet boundary conditions [2]. Functionals $b$ and $l$ depend on the inverse problem parameters $\mathbf{d}$. The variational problem (2) is equivalent with the minimization one (3), if $b$ is symmetric and positive definite (see e.g. [2])

$$
\left\{\begin{array}{l}
u \in u_{0}+V \\
E(u)=\frac{1}{2} b(u, u)-l(u) \longrightarrow \text { min. }
\end{array}\right.
$$

where $E(u)=\frac{1}{2} b(u, u)-l(u)$ is the functional of the total energy of the solution.

The Problem (2) may be approximated using the FEM on the finite dimensional subspace $V_{h, p} \subset V$

$$
\left\{\begin{array}{l}
u_{h, p} \in u_{0}+V_{h, p} \\
b\left(u_{h, p}, v_{h, p}\right)=l\left(v_{h, p}\right) \forall v_{h, p} \in V_{h, p}
\end{array} .\right.
$$

For a sequence of meshes generated by the self-adaptive $h p$ FEM code, for every coarse mesh, a coarse mesh space is a subset of the corresponding fine mesh space, $V_{h, p} \subset V_{h / 2, p+1} \subset V$.

An absolute relative FEM error utilized by the self-adaptive $h p$ FEM code is defined as the energy norm difference between the coarse and fine mesh solutions

$$
\operatorname{err}_{F E M}=\left\|u_{h, p}-u_{h / 2, p+1}\right\|_{E} .
$$

The inverse problem can be formulated as

$$
\text { Find } \hat{\mathbf{d}}:\left|J_{h, p}(\hat{\mathbf{d}})-J\left(\mathbf{d}^{*}\right)\right|=\lim _{h \rightarrow 0, p \rightarrow \infty} \min _{\mathbf{d}^{\mathbf{k}} \in \Omega}\left|J_{h, p}\left(\mathbf{d}^{\mathbf{k}}\right)-J\left(\mathbf{d}^{*}\right)\right|
$$

where $\mathbf{d}^{*}$ denotes exact parameters of the inverse problem (exact solution of the variational formulation for these parameters is well comparable with experiment data), $\mathbf{d}^{\mathbf{k}}$ denotes approximated parameters of the inverse problem, $\Omega$ is a set of all admissible parameters $\mathbf{d}^{\mathbf{k}}, J\left(\mathbf{d}^{*}\right)=E\left(u\left(\mathbf{d}^{*}\right)\right)$ is the energy of the 
exact solution $u\left(\mathbf{d}^{*}\right)$ of the variational problem (2) for exact parameters $\mathbf{d}^{*}$, $J_{h, p}\left(\mathbf{d}^{\mathbf{k}}\right)=E\left(u_{h, p}\left(\mathbf{d}^{\mathbf{k}}\right)\right)$ is the energy of the solution $u_{h, p}\left(\mathbf{d}^{\mathbf{k}}\right)$ of the approximated problem (4) for approximated parameters $\mathrm{d}^{\mathrm{k}}$.

Objective function error is defined as an energy difference between the solution of the approximated problem (44) for approximated parameter $\mathbf{d}^{\mathbf{k}}$ and the exact solution of the problem (2) for exact parameter $\mathbf{d}^{*}$ (assumed to be equal to the energy of the experiment)

$$
e_{h, p}\left(\mathbf{d}^{\mathbf{k}}\right)=\left|J_{h, p}\left(\mathbf{d}^{k}\right)-J\left(\mathbf{d}^{*}\right)\right| .
$$

In other words, the approximated parameter $\mathbf{d}^{\mathbf{k}}$ is placed into the approximated formulation (44), the solution of the problem $u_{h, p}\left(\mathbf{d}^{\mathbf{k}}\right)$ (which depends on $\mathbf{d}^{\mathbf{k}}$ ) is computed by FEM, and the energy of the solution $E\left(u_{h, p}\left(\mathbf{d}^{\mathbf{k}}\right)\right)$ is computed.

Lemma 1. $2\left[J_{h, p}\left(\mathbf{d}^{k}\right)-J_{h / 2, p+1}\left(\mathbf{d}^{k}\right)\right]=\left\|u_{h, p}\left(\mathbf{d}^{k}\right)-u_{h / 2, p+1}\left(\mathbf{d}^{k}\right)\right\|_{E}^{2}$

Proof: $2\left[J_{h, p}\left(\mathbf{d}^{k}\right)-J_{h / 2, p+1}\left(\mathbf{d}^{k}\right)\right]=2\left[E\left(u_{h, p}\left(\mathbf{d}^{k}\right)\right)-E\left(u_{h / 2, p+1}\left(\mathbf{d}^{k}\right)\right)\right]=$ $b\left(u_{h, p}\left(\mathbf{d}^{k}\right), u_{h, p}\left(\mathbf{d}^{k}\right)\right)-2 l\left(u_{h, p}\left(\mathbf{d}^{k}\right)\right)-b\left(u_{h / 2, p+1}\left(\mathbf{d}^{k}\right), u_{h / 2, p+1}\left(\mathbf{d}^{k}\right)\right)+$ $2 l\left(u_{h / 2, p+1}\left(\mathbf{d}^{k}\right)\right)=b\left(u_{h, p}\left(\mathbf{d}^{k}\right), u_{h, p}\left(\mathbf{d}^{k}\right)\right)-b\left(u_{h / 2, p+1}\left(\mathbf{d}^{k}\right), u_{h / 2, p+1}\left(\mathbf{d}^{k}\right)\right)+$ $2 l\left(u_{h / 2, p+1}\left(\mathbf{d}^{k}\right)-u_{h, p}\left(\mathbf{d}^{k}\right)\right)=b\left(u_{h, p}\left(\mathbf{d}^{k}\right), u_{h, p}\left(\mathbf{d}^{k}\right)\right)-$ $b\left(u_{h / 2, p+1}\left(\mathbf{d}^{k}\right), u_{h / 2, p+1}\left(\mathbf{d}^{k}\right)\right)+2 b\left(u_{h / 2, p+1}\left(\mathbf{d}^{k}\right), u_{h / 2, p+1}\left(\mathbf{d}^{k}\right)-u_{h, p}\left(\mathbf{d}^{k}\right)\right)=$ $b\left(u_{h / 2, p+1}\left(\mathbf{d}^{k}\right)-u_{h, p}\left(\mathbf{d}^{k}\right), u_{h / 2, p+1}\left(\mathbf{d}^{k}\right)-u_{h, p}\left(\mathbf{d}^{k}\right)\right)=$ $\left\|u_{h, p}\left(\mathbf{d}^{k}\right)-u_{h / 2, p+1}\left(\mathbf{d}^{k}\right)\right\|_{E}^{2}$

where $V_{h, p} \subset V_{h / 2, p+1} \subset V$ stand for the coarse and fine mesh subspaces.

Lemma 2. $e_{h / 2, p+1}\left(\mathbf{d}^{k}\right) \leq \frac{1}{2}\left\|u_{h / 2, p+1}\left(\mathbf{d}^{k}\right)-u_{h, p}\left(\mathbf{d}^{k}\right)\right\|_{E}^{2}+\left|J_{h, p}\left(\mathbf{d}^{k}\right)-J\left(\mathbf{d}^{*}\right)\right|$, where $e_{h / 2, p+1}\left(\mathbf{d}^{k}\right):=\left|J_{h / 2, p+1}\left(\mathbf{d}^{k}\right)-J\left(\mathbf{d}^{*}\right)\right|$.

Proof: $e_{h / 2, p+1}\left(\mathbf{d}^{k}\right)=\left|J_{h / 2, p+1}\left(\mathbf{d}^{k}\right)-J\left(\mathbf{d}^{*}\right)\right|=\mid J_{h / 2, p+1}\left(\mathbf{d}^{k}\right)-J_{h, p}\left(\mathbf{d}^{k}\right)+$ $J_{h, p}\left(\mathbf{d}^{k}\right)-J\left(\mathbf{d}^{*}\right)|\leq| J_{h / 2, p+1}\left(\mathbf{d}^{k}\right)-J_{h, p}\left(\mathbf{d}^{k}\right)|+| J_{h, p}\left(\mathbf{d}^{k}\right)-J\left(\mathbf{d}^{*}\right) \mid=$ $\frac{1}{2}\left\|u_{h / 2, p+1}\left(\mathbf{d}^{k}\right)-u_{h, p}\left(\mathbf{d}^{k}\right)\right\|_{E}^{2}+\left|J_{h, p}\left(\mathbf{d}^{k}\right)-J\left(\mathbf{d}^{*}\right)\right|$.

The objective function error over the fine mesh is limited by the relative error of the coarse mesh with respect to the fine mesh, plus the objective function error over the coarse mesh.

\section{Algorithm}

Lemma 2 motivates the following algorithm relating the inverse error with the objective function error. We start with random initial values of the inverse problem parameters

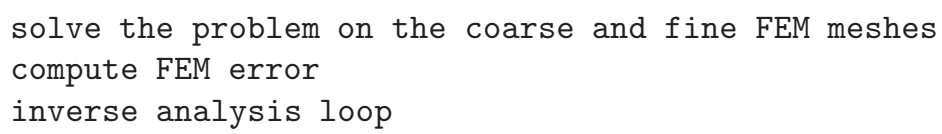

Propose new values for inverse problem parameters 


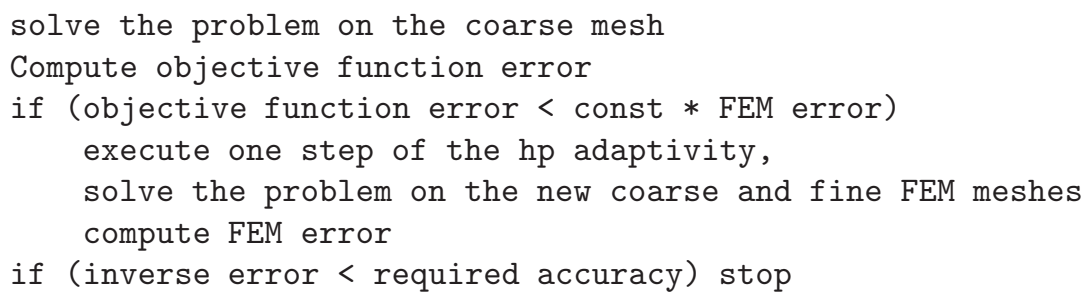

Inverse error estimation proven in Lemma $\mathbf{2}$ allows us to perform $h p$ adaptation in the right moment. If the objective function error is much smaller than the FEM error, the minimization of the objective function error does not make sense on current FE mesh, and the mesh quality improvement is needed.

\section{Step-and-Flash Imprint Lithography}

The above algorithm will be tested on the SFIL process simulation. The SFIL is a modern patterning process utilizing photopolymerization to replicate the topography of a template into a substrate. It can be summarized in the following steps, compare Fig. 2: Dispense - the SFIL process employs a template / substrate alignment scheme to bring a rigid template and substrate into parallelism, trapping the etch barrier in the relief structure of the template; Imprint - the gap is closed until the force that ensures a thin base layer is reached; Exposure - the template is then illuminated through the backside to cure etch barrier; Separate - the template is withdrawn, leaving low-aspect ratio, high resolution features in the etch barrier; Breakthrough Etch - the residual etch barrier (base layer) is etched away with a short halogen plasma etch; Transfer Edge the pattern is transferred into the transfer layer with an anisotropic oxygen reactive ion etch, creating high-aspect ratio, high resolution features in the organic transfer layer. The photopolymerization of the feature is often accompanied by the densification, see Fig. 2, which can be modeled by the linear elasticity with thermal expansion coefficient (CTE) [5]. We may define the problem: Find $u-$ displacement vector field, such that

$$
\left\{\begin{array}{c}
u \in V \subset\left(H^{1}(\Omega)\right)^{3} \\
b(u, v)=l(v) \forall v \in V
\end{array} .\right.
$$

where $V=\left\{v \in\left(H^{1}(\Omega)\right)^{3}: \operatorname{tr}(v)=0\right.$ on $\left.\Gamma_{D}\right\}, \Omega \subset R^{3}$ stands for the cubicshape domain, $\Gamma_{D}$ is the bottom of the cube and $H^{1}(\Omega)$ is the Sobolev space.

$$
b(u, v)=\int_{\Omega}\left(E_{i j k l} u_{k, l} v_{i, j}\right) d x ; \quad l(v)=\alpha \int_{\Omega} v_{i, i} d x .
$$

Here $E_{i j k l}=\mu\left(\delta_{i k} \delta_{j l}+\delta_{i l} \delta_{j k}\right)+\lambda \delta_{i j} \delta_{k l}$ stands for the constitutive equation for the isotropic material, where $\mu$ and $\lambda$ are Lame coefficients. The thermal expansion coefficient (CTE) $\alpha=\frac{\Delta V}{V \Delta T}$ is defined as a volumetric shrinkage of the edge barrier divided by $1 \mathrm{~K}$. 

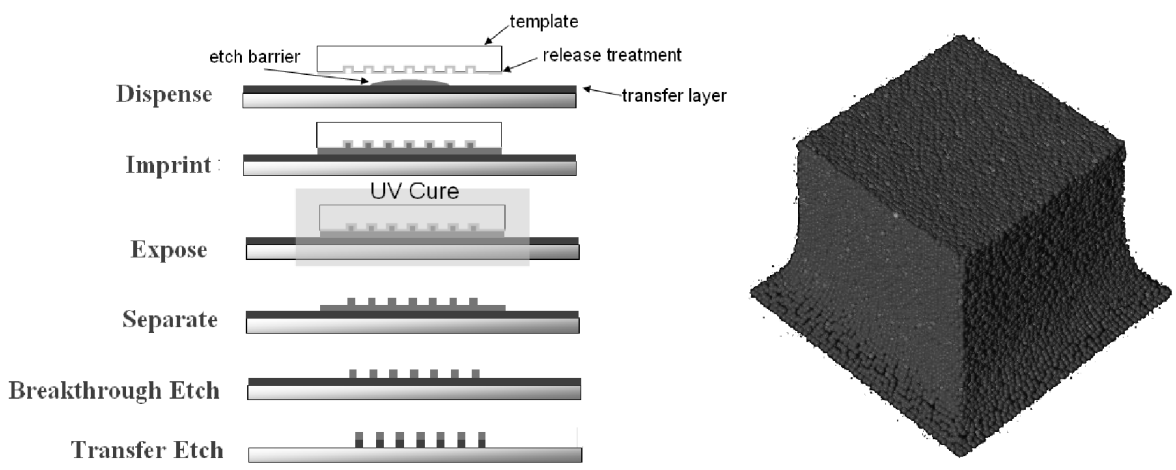

Fig. 2. Modeling of the Step-and-Flash Imprint Lithography process

\section{$6 \quad$ Numerical Results}

The proposed algorithm was executed for the problem of finding the proper value of the thermal expansion coefficient enforcing shrinkage of the feature comparable with experiments. The algorithm performed 43 iterations on the first optimal mesh (see the third picture in Fig. 1) providing $15 \%$ relative error of the direct problem solution. Then, the computational mesh was $h p$ refined to increase the accuracy of the direct solver. The inverse algorithm continued by utilizing $8 \%$ relative error mesh (see the fourth picture in Fig.1) for the direct problem. After 39 iterations the mesh was again $h p$ refined (see the fifth picture in Fig.1) to provide 5\% relative error of the direct problem solution. After 35 iterations of the inverse algorithm on the most accurate mesh the inverse problem was solved. The history of the (CTE) parameter convergence on the first, second and third optimal meshes is presented in Fig. 3.
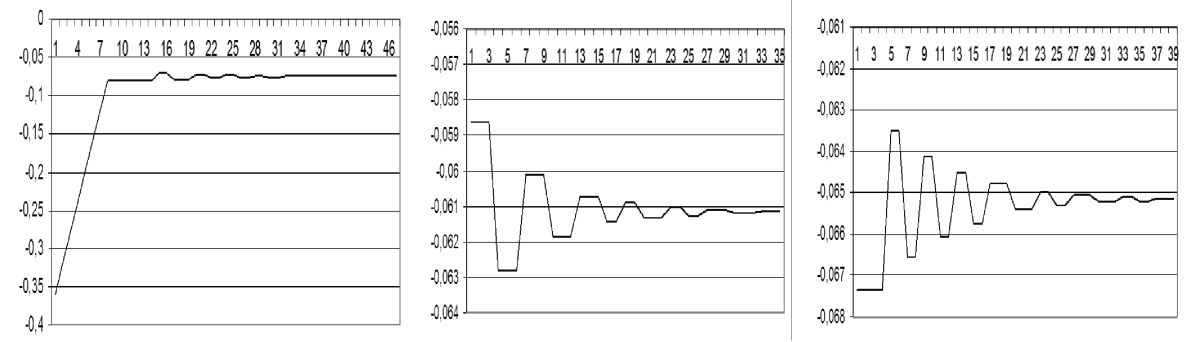

Fig. 3. History of convergence of CTE parameter on 3 meshes

We compared the total execution time equal to $0.1 s+43 \times 2 \times 0.1 s+1 s+39 \times$ $2 \times 1 s+10 s+35 \times 2 \times 10 s=8.7+79+710=797.7 s$ with the classical algorithm, where the inverse problem was solved on the most accurate FEM mesh from 
the beginning. The classical algorithm required 91 iterations to obtain the same result. The execution time of the classical algorithm was $10 s+91 \times 2 \times 10 s=$ $1830 \mathrm{~s}$.

This difference will grow when the inverse algorithm will look for more inverse problem parameters at the same time, since number of direct problem solutions necessary to obtain the new propositions of the inverse parameters will grow.

\section{The Molecular Static Model}

The energy of the experimental data $J\left(d^{*}\right)$ was estimated from the molecular static model, which provides realistic simulation results, well comparable with experiments [5]. During the photopolymierization, the Van der Waals bound between particles forming polymer chain are converted into a stronger covalent bounds. The average distance between particles is decreasing and the volumetric contraction of the feature occurs. In the following, a general equations governing the equilibrium configurations of the molecular lattice structure after the densification and removing of the template are derived.

Let us consider an arbitrary pair of bonded molecules with indices $\alpha$ and $\beta$ and given lattice position vector $p_{\alpha}=\left(\hat{x}_{\alpha}, \hat{y}_{\alpha}, \hat{z}_{\alpha}\right)$. The unknown equilibrium position vector of particle $\alpha$, under the action of all their intermolecular bonds, is denoted $x_{\alpha}=\left(x_{\alpha}, y_{\alpha}, z_{\alpha}\right)$, the displacements from the initial position in the lattice to the equilibrium position is represented by the vector $u_{\alpha}=x_{\alpha}-p_{\alpha}$. Let $\|\cdot\|$ denote the vector norm or length in $\mathbb{R}^{3}$, let $r_{\alpha \beta}=\left\|\mathbf{x}_{\beta}-\mathbf{x}_{\alpha}\right\|$ be the distance between particles $\alpha$ and $\beta$ in initial configuration. Then, the force $\mathbf{F}_{\alpha \beta}$, along the vector $x_{\beta}-x_{\alpha}$ is governed by the potential function $V\left(r_{\alpha \beta}\right)$,

$$
F_{\alpha \beta}=-\frac{\partial V\left(r_{\alpha \beta}\right)}{\partial r_{\alpha \beta}} \frac{x_{\beta}-x_{\alpha}}{\left\|x_{\beta}-x_{\alpha}\right\|} .
$$

where first term represents the magnitude and second term is the direction. If the indices of bonded neighboring particles of particle $\alpha$ are collected in the set $\mathcal{N}_{\alpha}$, then we obtain its force equilibrium by applying the following sum:

$$
\sum_{\beta \in \mathcal{N}_{\alpha}} F_{\alpha \beta}=-\sum_{\beta \in \mathcal{N}_{\alpha}} \frac{\partial V\left(r_{\alpha \beta}\right)}{\partial r_{\alpha \beta}} \frac{x_{\beta}-x_{\alpha}}{\left\|x_{\beta}-x_{\alpha}\right\|}=0 .
$$

The characteristics of the potential functions $\left\{V\left(r_{\alpha \beta}\right)\right\}_{\beta \in \mathcal{N}_{\alpha}}$ are provided by the Monte Carlo simulation [5]. The covalent bounds are modeled by spring forces

$$
F_{\alpha \beta}=C_{1} r+C_{2} \text {. }
$$

Spring like potential $V(r)$ is quadratic. The Van der Waals bounds are model by non-linear forces and the Lennard-Jones potentials

$$
V(r)=C_{\alpha \beta}\left[\left(\frac{\sigma_{\alpha \beta}}{r}\right)^{n_{\alpha \beta}}-\left(\frac{\sigma_{\alpha \beta}}{r}\right)^{m_{\alpha \beta}}\right] .
$$

where $r=\left\|x_{\beta}-x_{\alpha}\right\|$. 
The equilibrium equations are non-linear and the Newton-Raphson linearization procedure is applied to solve the system. The resulting shrinkage of the feature is presented in Figure 2.

\section{Conclusions and Future Work}

- The proper balance of errors of global optimization method and direct problem solvers allows for efficient speeding up the solution process of difficult inverse problems. The analytic relation among both errors is necessary.

- The relation between the objective function error and the relative error of the $h p$-adaptive FEM has been derived. The objective error was expressed as the energy difference between the numerical solution and experiment data.

- The strategy relating the convergence ratios of the inverse and direct problem solution has been proposed and successfully tested for searching value of the CTE parameter in the SFIL process. We obtained about 2.4 speedup in comparison to the solution without error balancing for the simple test example. The higher speedup may be expected for problems with larger dimension.

- The future work will include derivation of analytic relations between the $h p$ adaptive FEM error and objective function error defined in other ways. The possibilities of further speeding up of the solver will be tested by utilizing the parallel version of the $h p$-adaptive FEM codes [3].

Acknowledgments. The work reported in this paper was supported by Polish MNiSW grant no. 3 TO 8B 05529.

\section{References}

1. Ciarlet P., The Finite Element Method for Elliptic Problems. North Holland, New York (1994)

2. Demkowicz L., Computing with hp-Adaptive Finite Elements, Vol. I. Chapman \& Hall/Crc Applied Mathematics \& Nonlinear Science, Taylor \& Francis Group, Boca Raton London New York (2006)

3. Paszyński, M., Demkowicz, L., Parallel Fully Automatic hp-Adaptive 3D Finite Element Package. Engineering with Computers (2006) in press.

4. Paszyński, M., Szeliga, D., Barabasz, B. Macioł, P., Inverse analysis with 3D hp adaptive computations of the orthotropic heat transport and linear elasticity problems. VII World Congress on Computational Mechanics, Los Angeles, July 16-22 (2006)

5. Paszyński, M., Romkes, A., Collister, E., Meiring, J., Demkowicz, L., Willson, C. G., On the Modeling of Step-and-Flash Imprint Lithography using Molecular Statics Models. ICES Report 05-38 (2005) 1-26

6. Rachowicz, W., Pardo D., Demkowicz, L., Fully Automatic hp-Adaptivity in Three Dimensions. ICES Report 04-22 (2004) 1-52 